\title{
Multimodal imaging of temporal processing in typical and atypical language development
}

\author{
Ioulia Kovelman, ${ }^{1,2}$ Neelima Wagley, ${ }^{1}$ Jessica S. F. Hay, ${ }^{3}$ Margaret Ugolini, ${ }^{1,2,4}$ \\ Susan M. Bowyer, ${ }^{5}$ Renee Lajiness-O'Neill, ${ }^{2,6}$ and Jonathan Brennan ${ }^{7}$ \\ ${ }^{1}$ Department of Psychology, University of Michigan, Ann Arbor, Michigan. ${ }^{2}$ Center for Human Growth and Development, \\ University of Michigan, Ann Arbor, Michigan. ${ }^{3}$ Department of Psychology, University of Tennessee, Knoxville, Tennessee. \\ ${ }^{4}$ Department of Psychology, University of Massachusetts Amherst, Amherst, Massachusetts. ${ }^{5}$ Department of Neurology, \\ Henry Ford Hospital, Detroit, Michigan. ${ }^{6}$ Department of Psychology, Eastern Michigan University, Ypsilanti, Michigan. \\ ${ }^{7}$ Department of Linguistics, University of Michigan, Ann Arbor, Michigan
}

Address for correspondence: Ioulia Kovelman, Ph.D., Department of Psychology, University of Michigan, 530 Church Street, Ann Arbor, Ml 48109-1043. kovelman@umich.edu

\begin{abstract}
New approaches to understanding language and reading acquisition propose that the human brain's ability to synchronize its neural firing rate to syllable-length linguistic units may be important to children's ability to acquire human language. Yet, little evidence from brain imaging studies has been available to support this proposal. Here, we summarize three recent brain imaging (functional near-infrared spectroscopy (fNIRS), functional magnetic resonance imaging (fMRI), and magnetoencephalography (MEG)) studies from our laboratories with young Englishspeaking children (aged 6-12 years). In the first study (fNIRS), we used an auditory beat perception task to show that, in children, the left superior temporal gyrus (STG) responds preferentially to rhythmic beats at $1.5 \mathrm{~Hz}$. In the second study (fMRI), we found correlations between children's amplitude rise-time sensitivity, phonological awareness, and brain activation in the left STG. In the third study (MEG), typically developing children outperformed children with autism spectrum disorder in extracting words from rhythmically rich foreign speech and displayed different brain activation during the learning phase. The overall findings suggest that the efficiency with which left temporal regions process slow temporal (rhythmic) information may be important for gains in language and reading proficiency. These findings carry implications for better understanding of the brain's mechanisms that support language and reading acquisition during both typical and atypical development.
\end{abstract}

Keywords: language; literacy; rhythm; child; brain; autism spectrum disorder

\section{Introduction}

One of the greatest challenges in understanding human proficiency with language is determining what neural mechanisms support language and reading acquisition in early childhood. Early in life, infants must segment highly complex and continuous linguistic streams into sounds and syllablesthe building blocks of language. ${ }^{1,2}$ Later in development, children's ability to manipulate these phonological units becomes foundational for learning to read. ${ }^{3}$

Phonological units are comprised of relatively short segments (phonemes), such as the /b/ sound in the word "bat," as well as relatively longer units (syllables). ${ }^{4}$ Rhythmic characteristics of speech parallel, in an interesting way, rhythmic oscillatory patterns that characterize neuronal interactions, including both rapid gamma $(30-80 \mathrm{~Hz})$ and slow delta and theta $(1-8 \mathrm{~Hz})$ oscillations. ${ }^{5}$ Upon hearing speech, cortical neurons synchronize their firing rate to the frequencies of the linguistic input. ${ }^{4}$ In basic neuroscience, researchers have linked some instances of oscillatory synchrony to various types of environmental stimuli and brain responses during learning. ${ }^{6,7}$ Thus, researchers have put forth the idea that language-to-brain synchrony may also play a foundational role in language acquisition. ${ }^{4,8,9}$ 
Although there is accumulating evidence for such language-to-brain synchrony in adults, ${ }^{10}$ developmental neuroimaging evidence is lacking. Here, we report the findings of three studies from our labs where we address whether the brain's sensitivity to slow rhythmic syllable-length information (studies 1 and 2) and statistical information (study 3 ) relate to language and reading abilities in typically and atypically developing children.

At the beginning of language acquisition, babbling provides the first indicator of infant language production. At approximately 5 months old, infants begin to produce rhythmically alternating or repeating, meaningless syllables (e.g., ba-ba-ba). ${ }^{11}$ This milestone is considered universal as it emerges in infants exposed to any natural human language: infants exposed to a spoken language babble vocally whereas infants exposed to a sign language babble manually. ${ }^{12,13}$ Research into the temporal structure of babbling reveals that infants produce these rhythmically alternating units at a slow rate of about $3 \mathrm{~Hz}$ (i.e., approximately three syllables per second ${ }^{12}$ ) and that infants' jaw movements for vocal babbling and hand movements for manual babbling occur at the rate of about $1-2 \mathrm{~Hz} .^{13,14}$ These findings have led to the hypothesis that language-related regions of the brain might be sensitive to a selective range of temporal frequencies of around $1-3 \mathrm{~Hz}$ and that this sensitivity may help children discover syllables and words. ${ }^{13}$

Later in development, children's sensitivity to syllabic units is an early milestone for learning to read. Specifically, at the beginning of reading acquisition, children must learn to relate spoken language to print through explicit phonological awareness, which is the realization that spoken words are composed of phonological units that can be mapped onto orthographic representations. ${ }^{3}$ Children's sensitivity to larger phonological units of language, such as syllables and rhymes precedes and predicts learning to read cross-linguistically. ${ }^{3}$ A deficit in phonological awareness is thought to be the most common etiology of dyslexia ${ }^{3,15}$ and leads to life-long reading difficulties. Children with specific language impairment (SLI) demonstrate related deficits in phonological awareness, which is believed to lead to difficulties in learning to speak. ${ }^{16}$ Thus, in general, researchers have suggested a link between infants' sensitivity to linguistic units, such as syllables and phonemes, and later gains in language and reading acquisition across both typical and atypical development. ${ }^{17,18}$

Research employing nonlinguistic auditory tasks suggests that slow rhythmic linguistic units may drive the relationship between early sensitivity to syllables and phonemes and later language abilities in both typically and atypically (dyslexia and SLI) developing children..$^{8,19-22}$ For example, using experimental measures such as finger tapping to beats between 1 and $4 \mathrm{~Hz}^{22}$ or amplitude rise-time (ART) tasks ${ }^{8}$ researchers have found a relationship between individuals' slow temporal sensitivity and reading acquisition/dyslexia across multiple languages, including English, Spanish, Chinese, ${ }^{8,23,24}$ French, ${ }^{25}$ Finnish, ${ }^{26}$ and Hungarian. ${ }^{27}$ These behavioral data support the theoretical framework that the brain's sensitivity to slow rhythmically oscillating units of language (syllables and polysyllabic units, such as words) relates to, or possibly underlies, language acquisition in children. Yet, little direct neuroimaging evidence with children exists to support this developmental hypothesis. ${ }^{28}$

Thus, taking evidence from both language and reading acquisition studies, we suggest that early in life, infants' sensitivity to the slow temporal properties of the linguistic stream may help them identify and learn syllables and words of their native language. ${ }^{9}$ These syllabic units may then become available to be manipulated during phonological awareness tasks, an ability that is an important precursor for learning to read. ${ }^{21}$ Importantly, this hypothesis assumes that the brain's sensitivity to slow temporal modulations is related to language and reading acquisition in young children.

Neuroimaging data with adults suggest that the right auditory cortex has preferential sensitivity to slow temporal transitions. ${ }^{29-31}$ Furthermore, dysfunction in right-lateralized slow rhythmic processing may lead to language-based learning disabilities, especially dyslexia. ${ }^{21}$ Yet, neuroimaging research with typical young learners suggests that the left hemisphere is significantly more active than the right during language perception tasks, starting in early infancy. ${ }^{1,32}$ Moreover, reading improvement in children is also typically associated with changes in activation in left frontal and posterior temporal regions. ${ }^{33-36}$ Although these studies suggest a link between neural oscillations and reading acquisition, the precise connection between the brain bases of slow temporal processing in 
young children and language acquisition remains unclear.

Three recent neuroimaging studies from our laboratories have investigated these questions by exploring slow temporal perception and syllable processing in typically developing children and children diagnosed with autism spectrum disorder (ASD). We focused on two types of linguistic rhythms: (1) temporal properties of rhythmic syllable and phoneme alternations that are potentially universal across languages, ${ }^{29,37,38}$ and (2) vowel-toconsonant duration ratios that aid language segmentation differently across broad categories of syllable-timed (e.g., English) and stress-timed languages (e.g., Italian). ${ }^{39,40}$ Studies 1 and 2 focused on the potentially universal rhythmic properties of language, whereas study 3 also includes languagespecific rhythmic patterns as one of the variables. Each study used a different experimental approach and a different neuroimaging method-functional near-infrared spectroscopy (fNIRS), functional magnetic resonance imaging (fMRI), and magnetoencephalography (MEG) - with the goal of yielding converging multifaceted evidence to improve our understanding of how children's brain response to temporal information relates to language abilities and reading acquisition.

\section{Study 1-at the rhythm of language: fNIRS study of children's perception of rhythmic beats}

Studies of reading acquisition have shown that individuals with language-based learning difficulties, such as SLI and dyslexia, have difficulties finger tapping in tune with a metronome set to slow frequencies of about $1-3 \mathrm{~Hz} \cdot{ }^{19,22}$ It has been hypothesized that this temporal deficit is related to children's reduced sensitivity to syllabic units of language, necessary for both language and reading acquisition. ${ }^{21}$ In a recent study, ${ }^{41}$ we used fNIRS to better understand the neural bases of slow temporal processing by exploring children's brain responses to these "tappable" frequencies. Typically developing English readers (ages 6-9 years, $n=10$ ) were asked to listen passively to acoustic beats occurring at slow frequencies of $0.5,1.5$, and $3 \mathrm{~Hz}$ while we measured their brain activity in a block design. The goal of the study was to examine brain mechanisms at work when children perceive slow temporal modulations.
Two findings emerged: first, consistent with previously published findings with adult subjects, ${ }^{42}$ children showed greater overall right- than lefthemisphere activation during this task. Second, within the left hemisphere, children showed greater activation while listening to acoustic beats presented at $1.5 \mathrm{~Hz}$ relative to slower $(0.5 \mathrm{~Hz})$ and faster $(3 \mathrm{~Hz})$ frequencies. This effect was especially pronounced in the left superior temporal gyrus (STG) and parietal regions (for more details, see Ref. 41). Thus, these results confirm the overall right lateralization for slow temporal perception but also raise an important question as to why a subset of slow temporal frequencies might be privileged by the left hemisphere.

What might account for the privileged lefthemisphere response to $1.5 \mathrm{~Hz}$ rhythms? In Kovelman et al., ${ }^{41}$ we suggest that infants' discovery of language is supported by this sensitivity to a narrow set of slow-temporal frequencies, ideally suited for segmenting the linguistic stream into syllables and words. ${ }^{9}$ This hypothesis is supported by studies showing that infants' production of rhythmically alternating babbling is accompanied by hand movements at about $1.5 \mathrm{~Hz}$ for sign-exposed infants ${ }^{13}$ and mouth movements of about $1.8 \mathrm{~Hz}$ for auditory speech-exposed infants. ${ }^{14}$ Moreover, studies of vocal babbling have shown that at the onset of the babbling period, mouth aperture is wider and appears sooner on the right than the left side of the mouth, suggesting that infant babbling production is, at least initially, under control of the contralateral left hemisphere. ${ }^{43}$

These findings offer preliminary support for the idea that the left hemisphere might be sensitive to select a range of slow rhythmic modulations that help infants identify syllables and words in continuous auditory or visual linguistic stream and later help young readers actively manipulate these units of language for mapping them onto print.

\section{Study 2: amplitude rise-time perception}

We next used fMRI to study children's ART and phonological awareness (unpublished data). ${ }^{44}$ Slow temporal modulations of speech include rhythmically timed bursts of sound energy that occur at syllable and rhyme onsets. ${ }^{45}$ ART is the amount of time it takes for a sound to reach its peak volume. This acoustic transition typically accompanies the vowel portion of the syllable and thus contributes 
to the slow rhythmic properties found in the speech stream. ${ }^{21}$ Cross-linguistics literacy studies have shown that children's sensitivity to ART might be related to the development of phonological awareness and overall reading abilities in their native language. ${ }^{38}$ Yet, little is known about brain mechanisms underlying ART perception in young readers or how these relate to language and reading abilities. Thus, we used fMRI to explore the relationship between children's slow temporal perception, language and reading ability, and neural bases of ART perception.

Developmental research suggests that children with language-based learning disorders, such as dyslexia and SLI, show impaired performance on ART discrimination but maintain the ability to discriminate variations in intensity. ${ }^{22}$ Thus, we asked young readers (aged 6-12 years, $n=68$ ) to complete standardized measures of language, ${ }^{46}$ literacy, ${ }^{47}$ and literacy and ART $^{48}{ }^{48}$ A subset of the participants also completed ART Discrimination and Intensity Discrimination Control tasks modelled from Goswami et al., ${ }^{48}$ during fMRI in children aged 6-12 years $(n=15)$. The two tasks were similar in that children heard two different 800-ms pure tones (each tone was randomly associated with a different cartoon image) and indicated with a button press which sound (or cartoon animal) was softest (this judgment indicates which had the longest rise time or lower intensity). In the ART task, the standard tone (presented every trial) consisted of a 300-ms linear rise-time envelope, a 450-ms steady state, and a 50-ms linear fall time. The nonstandard tone varied in rise time from 19 to $29 \mathrm{~ms}$. The Intensity Discrimination task is considered to be perceptually simpler than the ART task, with sounds varying in intensity instead of rise time.

The two key preliminary findings of this study are as follows: first, consistent with previously published cross-linguistic findings, ${ }^{22,23,48}$ children's ART sensitivity threshold was negatively correlated with their language (phonology $r(67)=-0.27$; vocabulary $r(67)=-0.39, P<0.05)$ and reading (singleword reading $r(67)=0.31 ; P<0.05)$ abilities. Second, whole-brain correlation analyses revealed that children with better ART sensitivity threshold had less activity in the left STG during the ART task $(P \leq 0.001$ FWE cluster-level corrected, ET $=50)$. Similarly, children with better phonological awareness also had less activity in the left STG during the
ART task $(P \leq 0.001$ FWE cluster-level corrected, ET $=50)$. There were no significant correlations between children's performance on behavioral tasks of ART sensitivity, language, or literacy and brain activation during the Intensity Control task. The results suggest that children with better slow temporal and phonological abilities process slow temporal auditory modulations with less activity, consistent with greater automaticity, in the left STG region. These findings converge with those presented in study $1,{ }^{41}$ as discussed further in the conclusion section.

A recent intracranial electroencephalography (EEG) study with adult subjects provides further converging insights. ${ }^{49}$ Specifically, Morillon et al. suggest that although the right hemisphere might initially dominate processing of the slow temporal modulations of speech, it is the left hemisphere that is responsible for later integration of rapid/phonetic and slow/syllabic information within the slow temporal envelope, providing access to meaningful linguistic units. It is possible that by using hemodynamic techniques (fNIRS and fMRI) we have tapped into this second, higher-order process of temporal signal integration within the left hemisphere. Taken together, this evidence suggests that the left-hemisphere sensitivity to a narrow range of slow temporal modulations might play a significant role in successful language and reading acquisition. ${ }^{41,44,50}$

\section{Study 3-learning a new language}

Study 3 used MEG to investigate statistical learning in children with typical development or ASD (unpublished data). ${ }^{51}$ Research has shown that the brain's ability to synchronize its neuronal firing rate with syllabic units of language improves verbal understanding in adults, possibly because language-tobrain synchronization improves the brain's ability to parse continuous speech into syllabic units. ${ }^{31,52}$ This mechanism may also play an important role during language and reading acquisition in young children. ${ }^{9,21,28}$ Thus, in this study we have taken advantage of a statistical learning paradigm that emphasizes the importance of between-syllable predictability for finding words in continuous speech. Using MEG, we relate the brain's response to statistically predictive syllables with children's success rate of discovering words from fluent speech. 
Across languages, the transitional probability (TP) between syllables is higher within words and lower across word boundaries. The goal of this study was to explore the hypothesis that the brain's response to syllabic units of language contributes to a child's success in tracking statistical regularities in natural language. Specifically, we investigated the relationship between brain activity in response to syllable presentation and performance on a statistical learning task in children with ASD, who display impaired language acquisition and verbal communication skills (see Ref. 53 for a review), and their typically developing peers. MEG is well suited for recording millisecond-level neural activity, permitting precise alignment between neural oscillations and the occurrence of syllabic units within continuous speech. ${ }^{41,54}$

During MEG, children (aged 8-12 years old; ASD, $n=10$; neurotypical (NT), $n=9$ ) were familiarized with a 7 -min passage in a foreign language (Italian), produced in a lively register by a native Italian speaker. The familiarization corpus was modeled after the corpora used by Hay et al. ${ }^{57}$ and was grammatically correct in Italian. Importantly, Italian belongs to a rhythmically different class of languages as compared to English ${ }^{40}$ maximizing participants naiveté to the new language. The key manipulation was the insertion of four target words in the corpus. Two of the words had high internal predictability because their component syllables never occurred elsewhere in the corpus (high-TP words; $\mathrm{TP}=1.0$ ). The other two words had low internal predictability because their component syllables occurred may other times throughout the corpus (low-TP words; TP $=0.3$ ). Previously published research with similar stimuli has shown that infants as young as 8 months of age take advantage of statistical regularities to discover word-like sequences in artificial $^{2}$ and natural language materials. ${ }^{55,56}$ Furthermore, children's sensitivities to these statistical regularities are linked to subsequent word learning. ${ }^{57}$ Following familiarization, participants were asked to listen to a list of words that included both target words from the Italian corpus and novel words. For each word, children were asked to decide if it "sounded like it could be part of the new language." Preliminary analyses suggest that typically developing children displayed higher endorsement of both high-TP and low-TP words over novel words. In contrast, children with ASD displayed

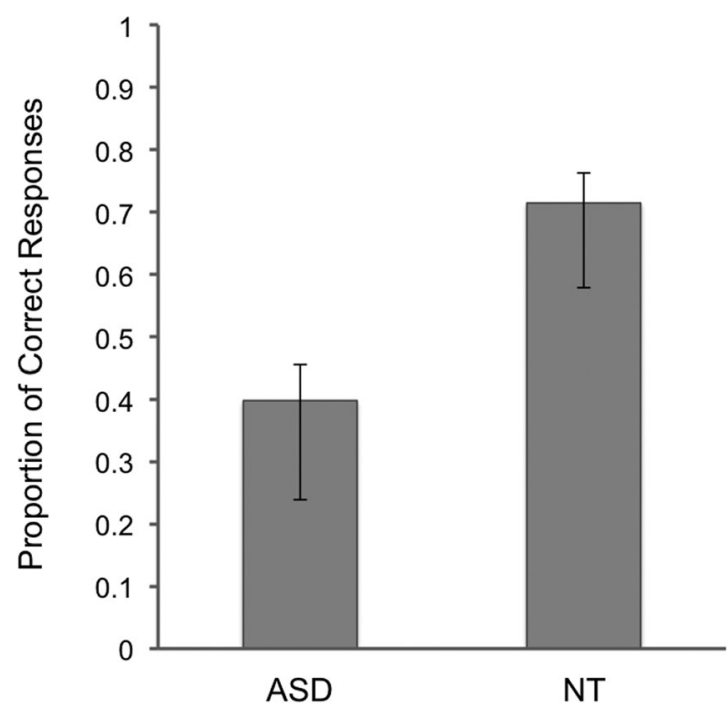

Figure 1. Proportion of correct responses indicating target words (averaged across high and low transitional probability words) relative to novel Italian words. Proportion correct was calculated out of 16 total trials from nine neurotypical (NT) children and a subset of eight children diagnosed with ASD, who completed the post-familiarization word test. Error bars indicate standard error of the mean.

on average a chance level of performance across all three word types (high-TP, low-TP, and novel words; Fig. 1).

Preliminary neuroimaging results also suggest differences between children with ASD and typically developing children. Specifically, participants' brain activity coherence (a measure of frequencydependent coordination of neuronal activity across brain regions ${ }^{58-61}$ ) during the familiarization phase was a significant predictor of whether the child had been diagnosed with ASD or was typically developing. Importantly, coherences measure during the familiarization phase showed improved outof-sample ASD diagnosis compared to coherences measured at rest (Fig. 2; see Ref. 62 for further details). Future MEG analyses will focus more on how the brain's response to syllables relates to language learning abilities and will include analyses of the brain's evoked response to low-TP versus high-TP words in children with ASD versus typically developing peers. ${ }^{51}$

Previously published research suggests that children with ASD show normal acquisition of high-TP words when those words occur in passages without variability in amplitude, duration, or pitch. ${ }^{63}$ Yet, they perform more poorly than typically developing 


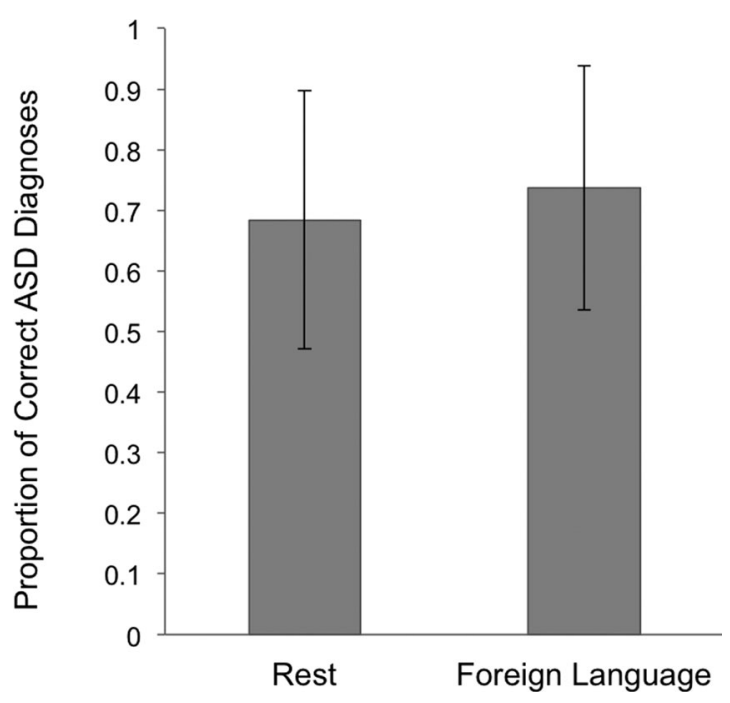

Figure 2. Proportion of correct diagnoses from a logistic classifier using coherence estimates from nine neurotypical children and 10 children diagnosed with ASD. MEG data from 10 min of eyes-open rest and $7 \mathrm{~min}$ of listening to a foreign language were band-pass filtered from 30 to $80 \mathrm{~Hz}$ and localized to a source grid spanning the gray matter of an age-matched pediatric template. Sources were grouped into 54 anatomical regions and pairwise coherence between regions was computed. Classifier features were the first two principle components defined over all coherences that showed a statistical trend for diagnostic group; predictive accuracy was tested using leave-one-out cross validation. Error bars show 95\% normal approximation binomial confidence intervals.

peers once these rhythmic cues are added. ${ }^{63}$ Similarly, fMRI findings have shown that typical learners show a decrease in brain activity (an index of habituation/learning) when listening to a statistically rich linguistic stream with prosodic cues relative to the one without it. ${ }^{64}$ In contrast, individuals with ASD did not show this decrease. Thus, it is possible that children with ASD have a reduced sensitivity to rhythmic cues that help identify statistically defined words in continuous speech. Further research is necessary to determine whether this deficit is also part of the etiology of language impairments in ASD and whether ASD children may have performed better if the new language belonged to the same rhythmic class (e.g., Dutch).

In summary, our prior work ${ }^{55-57}$ and present preliminary findings ${ }^{51}$ suggest that typically developing infants and children make use of statistical regularities to extract words embedded in a rhythmically rich linguistic stream. In contrast, converging evidence across prior ${ }^{63,64}$ and present findings suggest that children with ASD have difficulty extracting statistically defined words imbedded in a rhythmically rich linguistic stream and show nontypical brain responses during language learning tasks.

\section{General conclusion and implications for theories of sound and language acquisition}

The field has put forth a tantalizing hypothesis that a child's acquisition of language is supported by the brain's sensitivity to rhythmic modulations in speech, especially for slow rhythmic information carried by syllables and words $(1-8 \mathrm{~Hz})^{9,21,31}$ Preliminary data from our labs support this contention. First, Kovelman et al. ${ }^{41}$ suggest that a child's left hemisphere might play an important role in her/his ability to preferentially tune to temporal information linked to the first milestones of language ${ }^{11}$ and reading acquisition. ${ }^{21}$ Second, Ugolini et al. ${ }^{44}$ demonstrate that variations in children's language and reading ability might be linked to left-hemisphere sensitivity to slow temporal modulations. Finally, typically developing children, but not children with ASD, take advantage of syllablebased statistical regularities embedded in a rhythmically rich linguistic stream to extract words from a foreign language. The present findings both inform and extend the hypothesis that the brain's sensitivity to temporal information contributes to language and reading acquisition.

The theoretical model put forth by Zatorre et al. ${ }^{50}$ suggests that hemispheric asymmetries in temporal processing serve as the evolutionary basis for the brain's higher-order left-hemisphere specializations for rapid phonetic units, and righthemisphere specialization for music. Intracranial recordings show that, although the right hemisphere is indeed the first to respond to slow temporal language information, the left hemisphere nevertheless shows temporarily delayed responses to a select range of slow frequencies $(<5 \mathrm{~Hz})$. Hemispheric differences may aid in language processing by structuring rapid temporal information within meaningful syllable and word units. ${ }^{49}$ Functional NIRS and fMRI studies presented here likely captured this delayed left-hemisphere response, thus extending the Zatorre et al. ${ }^{50}$ framework to suggest the left hemisphere might have a delayed response to select slow temporal frequencies. 
The cortico-subcortical model of temporal processing put forth by Kotz and Schwartze et al. ${ }^{65}$ extends beyond the auditory modality and suggests that subcortical brain regions (i.e., cerebellum, basal ganglia, and thalamus) are sensitive to both visual and auditory sources of temporal information, which are then relayed to language-related cortical regions. Such a model helps explain convergent findings for our studies ${ }^{41,44}$ that aimed to tap into early language acquisition processes across natural sign and spoken languages. ${ }^{37}$

Finally, this work, which aims to link temporal processing with theories of language acquisition, is consistent with the recent framework proposed by Gervain and Mehler, ${ }^{39}$ suggesting that successful language acquisition rests on the complimentary and cooperative functioning of three major mechanisms: children's sensitivity to statistical regularities and rhythmic (vowel-based) properties of language, as well as social learning. The present findings support this perspective by highlighting the importance of the collaborative functioning of statistical, rhythmic, and social learning capabilities and its impairment in ASD. ${ }^{51}$ We further extend this hypothesis by suggesting that children's sensitivity to the rhythmic properties of language might comprise both sensitivity to overall language beat (e.g., $\mathrm{ART}^{44}$ as well as language-specific rhythm ${ }^{51}$ ) and that both of these abilities might be supported by left-hemisphere sensitivity to select slow rhythmic modulations. ${ }^{32}$

No single mechanism or process can account for the complexity of language acquisition. Nevertheless, the current evidence suggests that the brain's sensitivity to rhythmic properties found in speech merits further investigation as we work to uncover the mechanisms that support language and reading acquisition in children.

\section{Acknowledgments}

The authors thank Fondazione Mariani and the Neurosciences and Music Conference for affording us the opportunity to present and publish this work. The authors also thank the University of Michigan Departments of Psychology and Linguistics, Center for Human Growth and Development (CHGD), Functional MRI Laboratory, and MCubed project. Lastly, the authors thank the children and parents who contributed their time to these studies.

\section{Conflicts of interest}

The authors declare no conflicts of interest.

\section{References}

1. Petitto, L. et al. 2012. The "Perceptual Wedge Hypothesis" as the basis for bilingual babies' phonetic processing advantage: new insights from fNIRS brain imaging. Brain Language 121: 130-143.

2. Saffran, J.R., R.N. Aslin \& E.L. Newport. 1996. Statistical learning by 8-month-old infants. Science 274: 1926-1928.

3. Ziegler, J.C. \& U Goswami. 2005. Reading acquisition, developmental dyslexia, and skilled reading across languages: a psycholinguistic grain size theory. Psychol. Bullet. 131: 3 .

4. Morillon, B. et al. 2010. Neurophysiological origin of human brain asymmetry for speech and language. Proc. Natl. Acad. Sci. USA 107: 18688-18693.

5. Shalinsky, M.H. et al. 2002. Muscarinic activation of a cation current and associated current noise in entorhinal-cortex layer-II neurons. J. Neurophysiol. 88: 1197-1211.

6. Lakatos, P. et al. 2005. An oscillatory hierarchy controlling neuronal excitability and stimulus processing in the auditory cortex. J. Neurophysiol. 94: 1904-1911.

7. Womelsdorf, T. et al. 2005. Gamma-band synchronization in visual cortex predicts speed of change detection. Nature 439: 733-736.

8. Goswami, U. et al. 2011. Language-universal sensory deficits in developmental dyslexia: English, Spanish, and Chinese. J. Cogn. Neurosci. 23: 325-337.

9. Petitto, L.-A. 2005. "How the brain begets language: on the neural tissue underlying human language acquisition." In The Cambridge Companion to Chomsky. J. McGilvray, Ed.: 84-101. Cambridge: Cambridge University Press.

10. Giraud, A.-L. \& D. Poeppel. 2012. Cortical oscillations and speech processing: emerging computational principles and operations. Nat. Neurosci. 15: 511-517.

11. Petitto, L.A. et al. 2001. Language rhythms in baby hand movements. Nature 413: 35-36.

12. Petitto, L.A. \& P.F. Marentette. 1991. Babbling in the manual mode: evidence for the ontogeny of language. Science 251: 1493-1496.

13. Petitto, L.A. et al. 2001. Bilingual signed and spoken language acquisition from birth: implications for the mechanisms underlying early bilingual language acquisition. $J$. Child Language 28: 453-496.

14. Steeve, R.W. et al. 2008. Babbling, chewing, and sucking: oromandibular coordination at 9 months. J. Speech, Language, Hearing Res. 51: 1390-1404.

15. Shaywitz, S.E. \& B.A. Shaywitz. 2005. Dyslexia (specific reading disability). Biol. Psychiatry 57: 1301-1309.

16. Gathercole, S.E. 2006. Nonword repetition and word learning: the nature of the relationship. Appl. Psycholinguist. 27: 513-543.

17. Kuhl, P.K. et al. 2005. Early speech perception and later language development: implications for the "Critical Period." Language Learning Dev. 1: 237-264.

18. Molfese, D.L. 2000. Predicting dyslexia at 8 years of age using neonatal brain responses. Brain Language 72: 238-245. 
19. Corriveau, K.H. \& U. Goswami. 2009. Rhythmic motor entrainment in children with speech and language impairments: tapping to the beat. Cortex 45: 119-130.

20. Goswami, U. et al. 2002. Amplitude envelope onsets and developmental dyslexia: a new hypothesis. Proc. Natl. Acad. Sci. USA 99: 10911-10916.

21. Goswami, U., 2011. A temporal sampling framework for developmental dyslexia. Trends Cogn. Sci. 15: 3-10.

22. Thomson, J.M. \& U. Goswami. 2008. Rhythmic processing in children with developmental dyslexia: auditory and motor rhythms link to reading and spelling. J. Physiol.-Paris 102: 120-129.

23. Goswami, U. et al. 2013. Perception of patterns of musical beat distribution in phonological developmental dyslexia: significant longitudinal relations with word reading and reading comprehension. Cortex 49: 1363-1376.

24. Thomson, J.M., V. Leong \& U. Goswami. 2013. Auditory processing interventions and developmental dyslexia: a comparison of phonemic and rhythmic approaches. Reading Writing 26: $139-161$.

25. Muneaux, M. et al. 2004. Deficits in beat perception and dyslexia: evidence from French. NeuroReport 15: 1255-1259.

26. Hämäläinen, J. et al. 2008. Event-related potentials to pitch and rise time change in children with reading disabilities and typically reading children. Clin. Neurophysiol. 119: 100-115.

27. Surányi, Z. et al. 2009. Sensitivity to rhythmic parameters in dyslexic children: a comparison of Hungarian and English. Reading Writing 22: 41-56.

28. Obrig, H. et al. 2010. From acoustic segmentation to language processing: evidence from optical imaging. Frontiers Neuroenerg. 2: 13.

29. Poeppel, D. 2003. The analysis of speech in different temporal integration windows: cerebral lateralization as 'asymmetric sampling in time'. Speech Commun. 41: 245-255.

30. Boemio, A. et al. 2005. Hierarchical and asymmetric temporal sensitivity in human auditory cortices. Nat. Neurosci. 8: 389-395.

31. Poeppel, D. 2014. The neuroanatomic and neurophysiological infrastructure for speech and language. Curr. Opin. Neurobiol. 28: 142-149.

32. Peña, M. et al. 2003. Sounds and silence: an optical topography study of language recognition at birth. Proc. Natl. Acad. Sci. USA 100: 11702-11705.

33. Pugh, K.R. et al. 2001. Neurobiological studies of reading and reading disability. J. Commun. Disorders 34: 479-492.

34. Kovelman, I. 2011. "Neuroimaging methods." In Research Methods in Child Language: A Practical Guide. E. Hoff, Ed.: 43-59. Oxford: Wiley-Blackwell.

35. Kovelman, I. et al. 2012. Brain basis of phonological awareness for spoken language in children and its disruption in dyslexia. Cereb. Cortex 22: 754-764.

36. Lajiness-O'Neill, R., Y. Akamine \& S. Bowyer. 2008. Treatment effects of fast ForWord ${ }^{\circledR}$ demonstrated by magnetoencephalography (MEG) in a child with developmental dyslexia. Neurocase 13: 390-401.

37. Petitto, L.A. et al. 2004. Baby hands that move to the rhythm of language: hearingbabies acquiring sign languages babble silently on the hands. Cognition 93: 43-73.

38. Goswami, U. et al. 2014. Oscillatory 'temporal sampling' and developmental dyslexia: towards an over-arching theoretical framework. Front. Human Neurosci. 8: 904.
39. Gervain, J. \& J. Mehler. 2010. Speech perception and language acquisition in the first year of life. Ann. Rev. Psychol. 61: 191-218.

40. Ramus, F., M. Nespor \& J. Mehler. 1999. Correlates of linguistic rhythm in the speech signal. Cognition 73: 265-292.

41. Kovelman, I. et al. 2012. At the rhythm of language: brain bases of language-related frequency perception in children. NeuroImage 60: 673-682.

42. Zatorre, R.J. \& M. Schönwiesner. 2011. "Cortical speech and music processes revealed by functional neuroimaging." In The Auditory Cortex. J.A. Winer \& C.E. Schreiner, Eds.: 657-677. Springer.

43. Holowka, S. \& L.A. Petitto. 2002. Left hemisphere cerebral specialization for babies while babbling. Science 297: 1515.

44. Ugolini, M., N. Wagley, K. Ip, et al. In young readers, the left hemisphere supports the link between temporal processing and phonological awareness. In review.

45. Peelle, J.E. \& M.H. Davis. 2012. Neural oscillations carry speech rhythm through to comprehension. Front. Psychol. 3: 18.

46. Mitchell, J.-J. 2001. Comprehensive test of phonological processing. Assess. Effect. Interv. 26: 57-63.

47. Woodcock, R.W. 1998. Woodcock Reading Mastery Tests, Revised/Normative Update. American Guidance Service, Inc. Circle Pines, MN.

48. Goswami, U., D. Gerson \& L. Astruc. 2010. Amplitude envelope perception, phonology and prosodic sensitivity in children with developmental dyslexia. Reading Writing 23: 995-1019.

49. Morillon, B. et al. 2012. Asymmetric function of theta and gamma activity in syllable processing: an intra-cortical study. Front. Psychol. 3: 10.

50. Zatorre, R.J., P. Belin \& V.B. Penhune. 2002. Structure and function of auditory cortex: music and speech. Trends Cogn. Sci. 6: 37-46.

51. Wagley Neelima, M.U., J. Hay, S. Bowyer, et al. Learning a new language: MEG investigation of statistical learning in children with typical development and autism. In preparation.

52. Doelling, K.B. et al. 2014. Acoustic landmarks drive deltatheta oscillations to enable speech comprehension by facilitating perceptual parsing. Neuroimage 85: 761-768.

53. Groen, W.B. et al. 2008. The phenotype and neural correlates of language in autism: an integrative review. Neurosci. Biobehav. Rev. 32: 1416-1425.

54. Hämäläinen, M. et al. 1993. Magnetoencephalographytheory, instrumentation, and applications to noninvasive studies of the working human brain. Rev. Modern Phys. 65: 413.

55. Pelucchi, B., J.F. Hay \& J.R. Saffran. 2009. Learning in reverse: eight-month-old infants track backward transitional probabilities. Cognition 113: 244-247.

56. Pelucchi, B., J.F. Hay \& J.R. Saffran. 2009. Statistical learning in a natural language by 8 -month-old infants. Child Dev. 80: 674-685.

57. Hay, J.F. et al. 2011. Linking sounds to meanings: infant statistical learning in a natural language. Cogn. Psychol. 63: 93-106.

58. Elisevich, K. et al. 2011. An assessment of MEG coherence imaging in the study of temporal lobe epilepsy. Epilepsia 52: $1110-1119$. 
59. Bowyer, S.M. 2014. "Connectivity measurements for network imaging." In Electrophysiology and Psychophysiology in Psychiatry and Psychopharmacology. V. Kumari, P. Bob \& N.N. Boutros, Eds.: 315-330. Switzerland: Springer International Publishing.

60. Schoffelen, J.M. \& J. Gross. 2009. Source connectivity analysis with MEG and EEG. Human Brain Mapping 30: 18571865.

61. Groß, J. et al. 2002. The neural basis of intermittent motor control in humans. Proc. Natl. Acad. Sci. USA 99: 2299 2302.

62. Brennan, J., N. Wagley, M. Ugolini, et al. 2014. Neural coherence during natural story listening as a biomarker for
Autism. Presented at Society for the Neurobiology of Language Amsterdam, Netherlands.

63. Mayo, J. \& I.-M. Eigsti. 2012. Brief report: a comparison of statistical learning in school-aged children with high functioning autism and typically developing peers. J. Autism Dev. Disorders 42: 2476-2485.

64. Scott-Van Zeeland, A.A. et al. 2010. No neural evidence of statistical learning during exposure to artificial languages in children with autism spectrum disorders. Biol. Psychiatry 68: 345-351.

65. Kotz, S.A. \& M. Schwartze. 2010. Cortical speech processing unplugged: a timely subcortico-cortical framework. Trends Cogn. Sci. 14: 392-399. 